\title{
Die Bipolare Erkrankung im Kindes- und Jugendalter
}

E benso wie früher viele Forscher angenommen hatten, Kinder könnten nicht an Depressionen erkranken, glaubte man lange Zeit, eine bipolare Erkrankung könne nur im Erwachsenenalter auftreten (z.B. 2). Im Gegensatz hierzu stehen Ergebnisse retrospektiver Studien, die schon in den 20-er Jahren das Auftreten bipolarer Erkrankungen im Kindesalter nachweisen (9). In diesen Studien wurde gezeigt, dass 0-4\% Prozent aller bipolaren Erwachsenen schon im Kindesalter ( $<10$ Jahre) eine erste manisch-depressive Episode erlitten. Kraepelin berichtet sogar von einer sechsmonatigen manischen Episode eines fünfjährigen Kindes (9).

Heute wird die Prävalenz dieser Erkrankung im Kindes- und Jugendalter mit einem Wert von unter 0,1\% als relativ gering eingeschätzt (11). Man kann jedoch davon ausgehen, dass dieser Wert die tatsächliche Auftretenshäufigkeit bei weitem unterschätzt. Grund hierfür mag sein, dass in der kinderpsychiatrischen und -psychologischen Praxis das Beschwerdebild häufig fehlinterpretiert wird zugunsten einer im Allgemeinen häufiger diagnostizierten Erkrankung wie einer Aufmerksamkeitsdefizit-Hyperaktivitätsstörung (ADHS) oder einer Verhaltensstörung (5).

Eine umfassende (Differential-) Diagnostik ist also im Hinblick auf eine effektive Therapie unumgänglich. Untersuchungen haben gezeigt, dass bipolare Jugendliche eine signifikant höhere funktionale Beeinträchtigung aufweisen, eine erhöhte Komorbidität mit anderen Erkrankungen (Angststörungen, aggressive Verhaltensstörungen) und eine erhöhte Suizidgefährdung als rein unipolar depressive und psychisch gesunde Jugendliche der Vergleichspopulation (10).

\section{Diagnostik}

Zur Diagnostik bipolarer Erkrankungen im Kindes- und Jugendalter sollten die von Anthony und Scott (1) erarbeiteten zehn Diagnosekriterien herangezogen werden (11):

- Symptombild, das den klassischen Beschreibungen der bipolaren Erkrankung entspricht

- Vorkommen bipolarer Erkrankungen in der Familie

- schon frühe Neigung zu manisch-depressiven Reaktionsmustern

- mindestens einmaliges Vorkommen und Abklingen der Phasen

- zweiphasiger Verlauf (Anmerkung: dies ist jedoch kein zwingendes Kriterium. Störungen mit ausschließlich (hypo-)manischen Episoden werden ebenfalls zu den bipolaren Erkrankungen gerechnet, sind jedoch eher selten)

- Ausschluss äußerer Einflüsse auf den affektiven Zustand

- klinische Behandlungsbedürftigkeit, Leidensdruck

- abnorme, extravertierte Persönlichkeitsstruktur

- Ausschluss einer organischen Ursache oder einer Schizophrenie

- Absicherung der Diagnose durch den Verlauf.

Anmerkungen zu diesen Kriterien: Bei Erstmanifestation können natürlich noch nicht alle Kriterien erfüllt werden. Außerdem kann auch eine einzelne manische Episode auftreten, ohne dass eine bipolare Erkrankung vorliegt: Manische Symptome können auch Manifestationen einer schizophrenen Psychose oder hirnorganischen Ursache sein.

\section{Differentialdiagnostik}

Allgemein gilt: Differentialdiagnostisch ist die bipolare Erkrankung abzugrenzen von anderen affektiven Störungen, von Pubertäts- und Adoleszenzkrisen, von hirnorganischen Erkrankungen, von ADHS und Störungen des Sozialverhaltens (11).

Die differentialdiagnostische Abklärung kann u.a. durch genaue Evaluation der affektiven Beteiligung des Kindes erfolgen: Im Gegensatz zu einem Kind mit ADHS, einer Störung des Sozialverhaltens oder einer Pubertätskrise, kann das manische Kind (ebenso wie der manische Erwachsene) emotional nicht (oder kaum noch) erreicht werden. In den depressiven Phasen kann die Stimmung durch äußere Anlässe nicht aufgehellt werden.

Wichtig: ADHS und eine bipolare Erkrankung können auch gleichzeitig auftreten. Untersuchungen haben gezeigt, dass Kinder mit ADHS auch ein erhöhtes Risiko aufweisen, zusätzlich an einer bipolaren Störung zu erkranken (3).

\section{Verlauf der Erkrankung}

Stutte (12) beschreibt die Unterschiede zwischen der Ausprägung bipolarer Erkrankungen im Kindesund im Erwachsenenalter folgendermaßen:

In der Kindheit und Jugend kommt es generell zu häufigeren Phasenwechseln zwischen manischen und depressiven Episoden. Häufig treten depressive und manische Symptome auch gleichzeitig auf, wobei die Inhalte der jeweiligen Symptome geprägt sind vom jeweiligen Entwicklungsstand des Kindes/Jugendlichen. Manische Symptome sind bei Kindern eher durch starke Fluktuation der Symptomatik gekennzeichnet, die für Erwachsene typische Euphorie fehlt. Allgemein lassen sich im Kindesalter vor allem vegetative Funktionsstörungen beobachten, erst ab der Pubertät entwickelt sich die Symptomatik in Richtung des erwachsenen Krankheitsbildes (das bedeutet: je älter das Kind, desto ähnlicher wird der Krankheitsverlauf dem Verlauf des erwachsenen Krankheitsbildes).

Vorliegende Längsschnittstudien zeigen, dass bei ca. 20 bis $40 \%$ der Jugendlichen, die zunächst an einer schweren depressiven Episode erkranken, im Verlauf von fünf Jahren das Auftreten einer manischen Episode mit psychotischen Symptomen beobachtet werden kann. 70\% der 
Kinder, die an einer dysthymen Störung leiden, erleiden innerhalb von ca. zwei Jahren ihre erste schwere depressive Episode. Bei 13\% folgt dieser Episode eine manische Episode mit psychotischen Symptomen. Allgemein scheint zu gelten: Ein frühes Auftreten depressiver Symptome kann einen frühen Wechsel zu einer bipolaren affektiven Erkrankung zur Folge haben (11).

\section{- Therapie}

Eine sorgfältige Diagnostik ist wesentlich für eine effektive Therapie des Kindes/Jugendlichen. Je nach vorliegendem Störungsbild kommen unterschiedliche Therapiemaßnahmen zum Einsatz.

Während bei Störungen des Sozialverhaltens (etwa Störung des Sozialverhaltens mit oppositionellem, aufsässigem Verhalten) rein verhaltenstherapeutische Methoden (Kontingenzmanagement, Selbstinstruktionstraining, Token-Economy, etc.) hilfreich sein können, kann bei ADHS eine Kombination verhaltenstherapeutischer Interventionen mit medikamentöser Therapie (Methylphenidat o.ä.) sinnvoll sein.

Bei bipolaren Erkrankungen sollte in jedem Falle medikamentös interveniert werden (Stimmungsstabilisatoren wie Lithium, Carbamazepin, Valproinsäure, Lamotrigin, Kombinationsbehandlungen mit Antidepressiva/Neuroleptika) (siehe z.B. 6). Ergänzend zur medikamentösen Therapie kann die Durchführung einer Psychotherapie von großem Nutzen sein (6). Dies gilt ebenso für die Behandlung von bipolar erkrankten Kindern und Jugendlichen (11).

In depressiven Phasen können verhaltenstherapeutische Standardmethoden hilfreich sein (Aktivitätenaufbau, kindgemäße kognitive Umstrukturierung, etc). In manischen Phasen sind diese Methoden aufgrund mangelnder Einsichtsfähigkeit nicht/kaum nutzbar.

Psychoedukative Elemente (Rückfallprophylaxe, Erstellen eines Krisenplans, Erarbeiten von Bewältigungsmöglichkeiten der Erkrankung) sind bezüglich beider Pole der
Erkrankung wesentliche Bestandteile der therapeutischen Intervention. Je nach Alter und Entwicklungsstand des Kindes/Jugendlichen kann ein Einbezug der Eltern in den Therapieverlauf (v.a. bezüglich kognitiver Umstrukturierung, Erarbeiten von Belohnungssystemen, Psychoedukation) notwendig und sinnvoll sein. Als flankierende Maßnahmen kann ein Einbezug des schulischen und beruflichen Umfeldes erfolgen, ebenso der Versuch, die Stellung des Patienten im Kreise der Gleichaltrigen zu verbessern. (z.B. durch Einbezug des Kindes/des Jugendlichen in eine Gruppe).

\section{Fallgeschichte: \\ Der Fall Billy}

Ein Beispiel aus der Symptomsammlung des Kapitels „Treatable conditions parents should learn to recognize. Examples and Solutions“ in Hallowell, E. „When you worry about the child you love“.

Billy wurde im Alter von 15 Jahren aufgrund von immer häufiger werdenden Wutanfällen auffällig. In der Schule und zuhause verlor er zunehmendst die Beherrschung, konnte in der Schule nicht mehr stillsitzen, blieb mehrere Nächte in Folge wach.

Die Mutter, die nicht verstehen konnte, woher der Junge all diese Energie nahm, schaltete einen Arzt ein, der zusammen mit ihr Billy auf weitere Zeichen einer bipolaren Störung untersuchte: Größenideen oder „übergeschnappte“ Gedanken oder Verhalten, Ideenflucht, leichte Ablenkbarkeit, leichte Reizbarkeit, Schlaflosigkeit und Appetitverlust, Missbrauch von Alkohol oder Drogen, Suche nach maximaler Stimulation, z.B. mit lauter Musik, heftige Diskussionen oder Unterhalten, schnelles Fahren oder heftige Romanzen. Auch der depressive Pol der Erkrankung wurde bei Billy beobachtet: Antriebslosigkeit, Niedergestimmtheit, negative Gedanken, Hoffnungslosigkeit, Selbstwertprobleme.

Aufgrund einer rechtzeitig erfolgten Diagnose konnte Billy durch die richtige Behandlung geholfen werden, eine weitere Aggravation der Symptomatik wurde verhindert.

\section{Literatur:}

1. Anthony ] \& Scott P. Manic-depressive psychosis in childhood. Journal of Child Psychology and Psychiatry 1960; 1: 53-72

2. Barton-Hall M. Our present knowledge about manic-depressive states in childhood. Nerv Child 1952; 9: 319-325

3. Biederman J, Faraone $S$ et al. Attentiondeficit hyperacitivity disorder and juvenile mania: an overlooked comorbidity? J Am Acad Child Adolesc Psychiatry 1996; 35 (8): 997-1008

4. Bradley C. Definition of childhood in psychiatric literature. American Journal of Psychiatry 1937; 94: 33-36

5. Carlson GA, Annotation: child and adolescent mania- diagnostic considerations. Journal of Child Psychology and Psychiatry 1990; 31: 331-341

6. Grunze H, Walden J. Die Behandlung bipolarer Störungen. In: Die Bipolaren Störungen. Manisch-depressive Erkrankungen. Ratgeber für Betroffene und ihre Angehörige. Stuttgart, Georg Thieme Verlag, 2003: 17-49

7. Hallowell E. Treatable conditions parents should learn to recognize. In: Hallowell $E$. When you worry about the child you love. New York, Fireside, 1997: 41-42

8. Hallowell E. Mad: When Children act up. In: Hallowell E. When you worry about the child you love. New York, Fireside, 1997: 115-154

9. Kraepelin E. Manic-depressive Insanity and Paranoia. Edinburgh, Livingstone, 1921 10. Lewinsohn P, Klein D \& Seeley J. Bipolar disorders in a community sample of older adolescents: prevalence, phenomenology, comorbidity, and course. Journal of Am Acad Child Adolesc Psychiatry 1995; 34 (4): 454-463

11. Remschmidt H. Affektive Störungen. In: Remschmidt H. (Ed.). Kinder- und Jugendpsychiatrie - Eine praktische Einführung. Stuttgart, Thieme, 2000: 196-206

12. Stutte H. Endogen-phasische Psychosen im Kindesalter. Acta Paedopsychiatrica 1963; 30: 34-42

13. Warnke A, Jans T \& Remschmidt H. Bipolare affektive Störung. In: Harrington RC. Kognitive Verhaltenstherapie bei depressiven Kindern und Jugendlichen. Göttingen, Hogrefe, 2001

\section{Korrespondenzadresse:}

Dipl.-Psych. Ingeborg Schweiß Psychiatrische Klinik und Poliklinik der LMU München Nußbaumstraße 7

80336 München

Deutsche Gesellschaft für Bipolare Störungen e.V. (DGBS e.V.)

Postfach 920249

21132 Hamburg

Tel.: 04085408883

E-Mail:info@dgbs.de

Internet: www.dgbs.de 